\title{
Face Tracking and Head Pose Estimation using Convolutional Neural Networks
}

\author{
Stylianos Asteriadis*, KostasKarpouzis ${ }^{\dagger}$ Stefanos Kollias ${ }^{\ddagger}$ \\ Image, Video, Multimedia Lab, National Technical University of Athens, Greece
}

\section{Introduction}

In applications where face orientation is necessary, but in unpretending environments in terms of lighting, equipment, resolution, etc, employing local tracking techniques would usually fail to give accurate results, regarding the issue of head pose estimation. However, in a similar manner, holistic techniques require the face to be well aligned with the training data. This pre-assumes correct and accurate face tracking, which is also a challenging issue. Here, we propose a face tracker, adjusted to each person's face chrominance values, and learnt online. Based on the face bounding box, Convolutional Neural Networks (CNNs) are employed, in order to calculate face orientation. CNNs are ideal for cases where a lot of distortions exist in the data, and the proposed architecture only utilizes subsets of classifiers, excluding those corresponding to rotation angles far from the current.

\section{Face Tracking}

Face is initially detected using the detector of Viola-Jones [Viola and Jones 2001]. Subsequently, a sample area $C_{\text {skin }}$ of face is used for each person; we used the saturation values of this area, and the saturation values of face pixels $C_{f p}$ in subsequent frames are expected to be within certain limits (defined by threshold $T$ )with regards to the mean saturation value $s_{M}$ of $C_{s k i n}$. The binary image of these pixels $C_{f p}$ is formed, followed by binary opening. The threshold $T$ is automatically selected for each user, at the detection step, according to the hypothesis below: it is expected that, at the first frame, the amount of pixels with saturation values close to the mean of $C_{\text {skin }}$ is close to the amount of pixels that account for the real face region. Thus, the threshold that gives amount of pixels closer to the expected face size, is the one kept.

To reduce the number of candidate facial pixels, the rules defined in [Jure Kovac and Solina 2003] are used, in order for skin clusters in $R G B$ colorspace to be built and the resulting binary map $C_{f} p$ is combined using logical $A N D$ with $C_{s} p$. Finally, the proposed method uses connected component labelling and chooses the largest component as the final face region.

\section{Convolutional Neural Networks}

In a generic image recognition problem, an image is used as input in the first layer of a CNN [LeCun et al. 1990] and it is convolved with a series of filters in the second layer $(C 1)$, resulting in feature maps. In the third layer $(S 2)$, the feature maps are subsampled. Further such levels may follow. This architecture guarantees limited number of free parameters, automatic extraction of complex and spatial features and robustness to distortions. The architecture employed in the proposed scheme, is a 6-layer convolutional neural network, with the layers being in the order $C 1$ (6 maps), $S 2, C 3$ (6 maps), $C 4$ (80 maps), F5 (10 neurons), F6 (output -2 neurons), which is a 2 -element vector. The convolutional layers use $7 \times 7$ kernels, while the sub-sampling layer uses a downscale factor of 2 . Inputs are $32 \times 32$ normalized face imagettes.

\footnotetext{
*e-mail: stiast@image.ntua.gr

†e-mail: kkarpou@image.ntua.gr

‡e-mail: stefanos@cs.ntua.gr
}

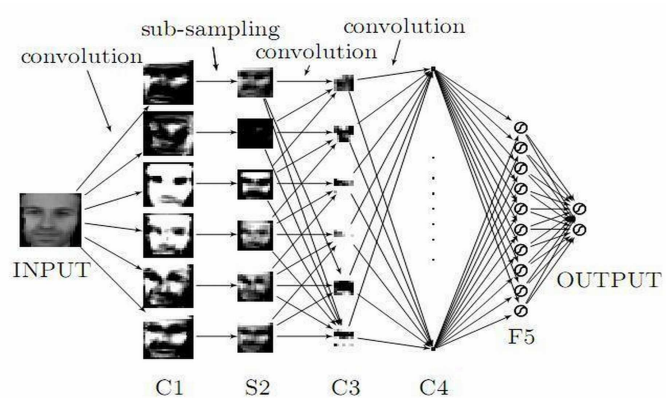

Figure 1: Employed CNN architecture.

\subsection{Training Procedure and Results}

For training, we created a pose space consisting of classes centered at pitch angles $\left\{-60^{\circ}, 0,60^{\circ}\right\}$ and yaw angles $\left\{-90^{\circ},-45^{\circ}, 0,45^{\circ}, 90^{\circ}\right\}$. We trained one $\mathrm{CNN}$ for each combination of neighboring classes, resulting in a total of 38 classifiers. Using yaw and pitch information from the previous frame, all $n$ networks considered include the class $C_{c}$ whose center is closer to the the yaw and pitch values of the previous frame. In this way, only a subset of CNNs are used at each frame, constituting the system faster and more reliable, as the possibility of erroneous classification is reduced. The final estimate of the yaw (or pitch) angle is done by employing regression models including elements of the differences of outputs, as well as the centre of class $C_{c}$.

The method has been tested on the Boston University Face dataset: and errors regarding yaw and pitch were $5.6^{\circ}$ and $4.7^{\circ}$, respectively.

\section{Acknowledgements}

This work was funded by ICT-Project 'Siren', (under contract FP7ICT-258453)

\section{References}

Jure Kovac, P. P., AND Solina, F. 2003. Human skin colour clustering for face detection. In IEEE International Conference on Computer as a Tool, vol. 2.

LeCun, Y., Boser, B., Denker, J. S., Henderson, D., Howard, R. E., Hubbard, W., AND JaCKel, L. D. 1990. Handwritten digit recognition with a back-propagation network. In Advances in Neural Information Processing Systems, Morgan Kaufmann, 396-404.

Viola, P. A., AND Jones, M. J. 2001. Rapid object detection using a boosted cascade of simple features. In Conference on Computer Vision and Pattern Recognition, vol. 1, 511-518. 